\title{
Endovascular Therapy in Strokes with ASPECTS 5-7 May Result in Smaller Infarcts and Better Outcomes as Compared to Medical Treatment Alone
}

\author{
Ali Reza Noorian Srikant Rangaraju Chung-Huan Sun Kumiko Owada \\ Fadi Nahab Samir R. Belagaje Aaron M. Anderson Michael R. Frankel \\ Raul G. Nogueira \\ Department of Neurology, Marcus Stroke and Neuroscience Center, Grady Memorial \\ Hospital, Emory University School of Medicine, Atlanta, Ga., USA
}

\section{Key Words}

Stroke $\cdot$ Endovascular intervention $\cdot$ CT scan

\begin{abstract}
Introduction: Intra-arterial therapy (IAT) for large vessel occlusion strokes (LVOS) has been increasingly utilized. The benefit of IAT in patients with midrange Alberta Stroke Program Early Computed Tomography Score (ASPECTS) remains to be established. Materials and Methods: This was a retrospective analysis of LVOS with ASPECTS 5-7 treated with IAT ( $\mathrm{n}=$ 86) or medical therapy alone (intravenous tissue plasminogen activator; $n=15$ ) at two centers from 2009 to 2012. Definitions were as follows: symptomatic intracranial hemorrhage = any parenchymal hematoma; successful reperfusion = $\mathrm{mTICI} \geq 2 \mathrm{~b}$; good and acceptable outcomes $=90$-day mRS $0-2$ and $0-3$, respectively. Final infarct volumes (FIV) were calculated based on 24-hour CT/MRI scans. Results: Mean age (67 \pm 14 vs. $67 \pm 19$ years) and baseline NIHSS ( $20 \pm 5$ vs. $20 \pm 6$ ) were similar in the two groups. Successful reperfusion was achieved in $58(67 \%)$ IAT patients. Symptomatic and asymptomatic intracranial hemorrhage occurred in $9(10 \%)$ and 31 (36\%) IAT patients, respectively. The proportion of 90 -day good and acceptable outcomes was 20 (17/86) and 33\% (28/86), respectively. Successful IAT reperfusion was associated with smaller FIV ( $p=0.015)$ and higher rates of good $(p=0.01)$ and acceptable $(p=0.014)$ outcomes. There was a strong trend towards a higher hemicraniectomy requirement in medically as compared to endovascularly treated patients ( $20 \mathrm{vs.} 6 \% ; p=0.06)$ despite similar in-hospital mortality. The median FIV was significantly lower with IAT versus medical
\end{abstract}

The content of this paper was presented at the 11th International Stroke Summit, Nanjing, 2015.

Raul G. Nogueira, MD 
therapy [80 $\mathrm{ml}$ (interquartile range, 38-122) vs. $190 \mathrm{ml}(121-267) ; \mathrm{p}=0.015]$. Conclusions: Despite a relatively low probability of achieving functional independence, IAT in LVOS patients with ASPECTS 5-7 appears to result in lower degrees of disability and may lessen the need for hemicraniectomy. Therefore, it may be a reasonable option for patients and families who favor a shift from severe to moderate disability.

(C) 2015 S. Karger AG, Base

\section{Introduction}

The benefit of acute stroke care is directly related to timely recanalization of the occluded cerebral artery with the ultimate goal of restoring penumbral tissue function and improving clinical outcomes. However, only $15 \%$ of the stroke patients reached an emergency room within the 3-hour window, and despite the extension of the time window to $4.5 \mathrm{~h}$, only about $10 \%$ of all ischemic stroke patients currently receive intravenous recombinant tissue plasminogen activator (IV rt-PA) [1, 2]. Moreover, the rates of recanalization with IV rt-PA for large vessel occlusion strokes (LVOS) range from only 4-35\% for internal carotid artery (ICA) occlusion to $30-77 \%$ for middle cerebral artery (MCA) occlusion and 5-53\% for basilar occlusions [3-7]. Nonetheless, successful reperfusion remains one of the strongest predictors of good outcomes after ischemic stroke [8]. Indeed, it has been recently established that untreated LVOS patients presenting with moderate-to-severe deficits [National Institutes of Health Stroke Scale (NIHSS) score $\geq 10$ ] have less than one in four chances of functional independence and greater than one in three chances of death [9]. Endovascular therapy has therefore emerged as a promising strategy in the care of severe ischemic strokes patients who are refractory to or ineligible for intravenous thrombolysis, with reperfusion rates of $50-86 \%$ [10].

Large pretreatment infarct core size on noncontrast CT scan has been associated with higher rates of intracranial hemorrhage (ICH) and worse clinical outcomes [11]. The Alberta Stroke Program Early Computed Tomography Score (ASPECTS) is a 10-point scale to score early ischemic changes in ten defined regions in the MCA territory with a total score ranging from 10 (no evidence of early ischemia) to 0 (all 10 regions in the hemisphere show early ischemic changes). Patients with ASPECTS 8-10 appear to have the best clinical outcomes with reperfusion therapy, while patients with baseline ASPECTS $\leq 4$ do not seem to benefit and may actually be harmed by treatment [12]. Although patients with ASPECTS 5-7 may benefit from IV rt-PA within $3 \mathrm{~h}$ [13], the effects of endovascular therapy in patients with ASPECTS 5-7 remain to be established. In this study, we sought to determine the clinical outcomes in patients with LVOS who presented with ASPECTS of 5-7 and were treated with medical management alone and/or endovascular therapy.

\section{Methods}

Subjects

After Institutional Review Board approval, a retrospective chart review was performed to identify all consecutive patients with LVOS who underwent intra-arterial therapy (IAT) at a Level 1 Trauma Center (Grady Memorial Hospital, where endovascular treatment was aggressively pursued) or who were medically treated (including IV t-PA if eligible) at a tertiary referral center (Emory University Hospital, where endovascular stroke treatment was uncommonly performed) from 2009 to 2012. Baseline characteristics were collected from hospital records including demographics, vascular risk factors, radiographic variables, time to treatment and requirement for hemicraniectomy. LVOS were defined as strokes due to occlusion involving the intracranial ICA or MCA M1 and/or M2 segments (with or without concomitant extracranial ICA occlusion) 
Noorian et al.: Endovascular Therapy in Strokes with ASPECTS 5-7 May Result in

Table 1. Baseline characteristics

\begin{tabular}{lccc}
\hline & $\begin{array}{c}\text { IAT group } \\
(\mathrm{n}=86)\end{array}$ & $\begin{array}{c}\text { Medically treated } \\
\text { group (n=15) }\end{array}$ & p value \\
\hline Age, years & $66.9 \pm 14.4$ & $66.8 \pm 19.3$ & 0.989 \\
Males & $44(51)$ & $5(33)$ & 0.202 \\
Diabetes mellitus & $21(24)$ & $4(27)$ & 0.872 \\
Hypertension & $58(67)$ & $9(60)$ & 0.568 \\
Atrial fibrillation & $25(29)$ & $8(53)$ & 0.069 \\
Currently smoking & $19(22)$ & $2(13)$ & 0.429 \\
NIHSS score & $19.8 \pm 5.0$ & $19.5 \pm 6.1$ & 0.869 \\
IV rt-PA use & $48(56)$ & $6(40)$ & 0.27 \\
ASPECTS & $6[6-7]$ & $6[5.5-7]$ & 0.450 \\
LKN to pretreatment CT, min & $247[184-345]$ & n.a. & 0.841 \\
LKN to reperfusion, min & $386[314-489]$ & $7(47)$ & \\
Vessel ICA & $55(64)$ & $3(20)$ & \\
M1 & $8(9)$ & $5(33)$ & 0.299 \\
M2 & $23(27)$ & & \\
\hline
\end{tabular}

Data are presented as $n(\%)$, median $[\mathrm{IQR}]$ or mean \pm SD. n.a. = Not available.

identified by CT, MR or conventional angiography. ASPECTS were calculated for all the patients based on a standard protocol [14]. Only patients with ASPECTS 5-7 on pretreatment CT were included in the current analysis.

\section{Outcome Measures}

Successful reperfusion was defined as modified TICI $2 \mathrm{~b}$ or greater [15]. A 'good' clinical outcome was defined as a 90-day modified Rankin Scale (mRS) score of 0-2, while 'acceptable' outcome was defined as an $\mathrm{mRS}$ score of $0-3$. Infarct volume was calculated by summation of the regions of interest outlining the final infarct on a CT or MRI obtained within $24 \mathrm{~h}$ after the procedure. Infarct volume $<70 \mathrm{ml}$ was considered favorable in the analysis [16]. Long-term outcomes (e.g. 90-day mRS) were only available for IAT patients.

\section{Statistical Analysis}

Continuous variables were reported as mean \pm standard deviation or as median \pm interquartile range (IQR). Categorical variables were reported as proportions. Differences between proportions were assessed by the $\chi^{2}$ test ( $\alpha=0.05$ for significance). As an attempt to demonstrate the effects of successful reperfusion in LVOS patients with baseline ASPECTS 5-7, we compared the rates of good outcomes, acceptable outcomes and mortality as well as the final infarct volumes amongst recanalized versus nonrecanalized IAT patients. In addition, we compared the final infarct volumes and rates of hemicraniectomy amongst the IAT and medically treated patients. Statistical analysis was performed using SPSS 20 and Microsoft Excel.

\section{Results}

\section{Baseline and Procedural Characteristics}

A total of 101 patients were identified, with 86 patients in the IAT group [mean age: 67 \pm 14 years; NIHSS: $20 \pm$ 5; ASPECTS 5: 16 (19\%) patients; ASPECTS 6: 28 (33\%) patients; ASPECTS 7: 42 (49\%) patients] and 15 patients in the medically treated group [mean age: 67 \pm 19; NIHSS: $20 \pm$ 6; ASPECTS 5: 4 (27\%) patients; ASPECTS 6: 5 (33\%) patients; ASPECTS 7: $6(40 \%)$ patients]. There were no significant differences in the baseline characteristics between the two groups (table 1). Forty-eight patients of the IAT group (56\%) and 6 of the medically treated group (40\%) received IV rt-PA. The median (IQR) times from last known 
Fig. 1. Association between the degree of reperfusion (shown by mTICI score) with good (a; 90day mRS 0-2: mTICI $0-2 \mathrm{a}=3.7 \%$ vs. mTICI $2 b-3=31 \%$; $p=0.01$ ) and acceptable (b; 90-day mRS 0-3: mTICI $0-2 \mathrm{a}=14.8 \%$ vs. mTICI $2 \mathrm{~b}-3=44.8 \%$; $\mathrm{p}=0.014$ ) functional outcomes.

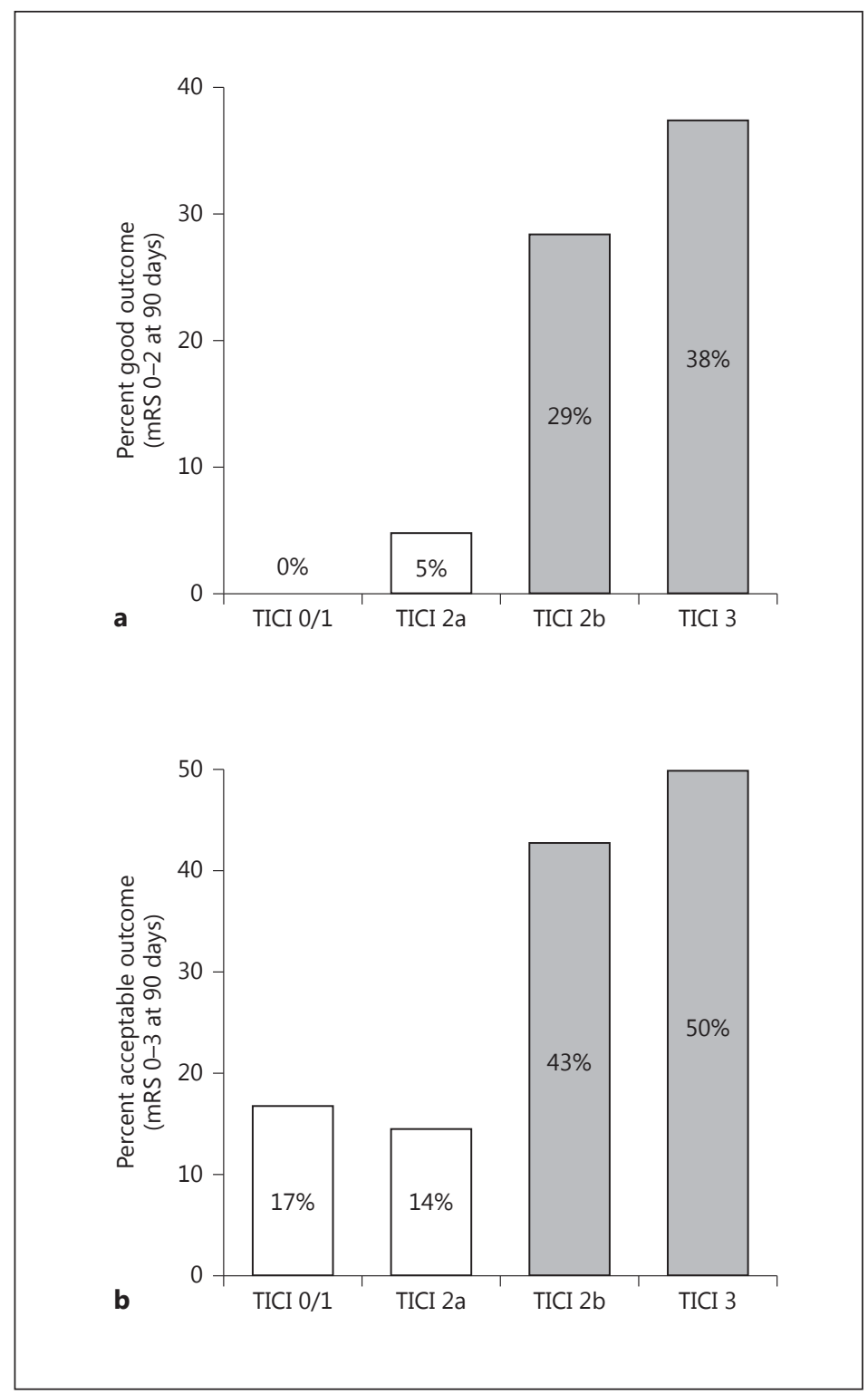

normal (LKN) to groin puncture were 300 (228-400) min for the whole IAT group and 350 (269-440) min for the IAT subgroup not treated with rt-PA. The first device used in endovascular thrombectomy was Solitaire in 35 patients (41\%), Merci in 24 patients (28\%), Penumbra in 22 patients $(25 \%)$ and other in 5 patients $(6 \%)$.

\section{Outcomes in the Endovascular Treatment Group}

In the IAT group, successful reperfusion [modified thrombolysis in cerebral infarction (mTICI) score $\geq 2 \mathrm{~b}$ ] was achieved in 58 patients (67\%; mTICI 0-1: 6; mTICI 2a: 22; mTICI 2b: 42; mTICI 3: 16). Seventeen IAT patients (20\%) achieved a good clinical outcome, while 28 (33\%) achieved an acceptable outcome. Patients with higher grades of reperfusion had higher likelihood of achieving better clinical outcomes (fig. 1). Optimal degrees of reperfusion (mTICI $\geq 2 \mathrm{~b}$ ) were more likely associated with good (31 vs. $3.7 \% ; \mathrm{p}=0.01$ ) and acceptable (44.8 vs. $14.8 \% ; \mathrm{p}=0.014$ ) outcomes as compared to suboptimal or no reperfusion (mTICI $0-2 \mathrm{a}$ ). 


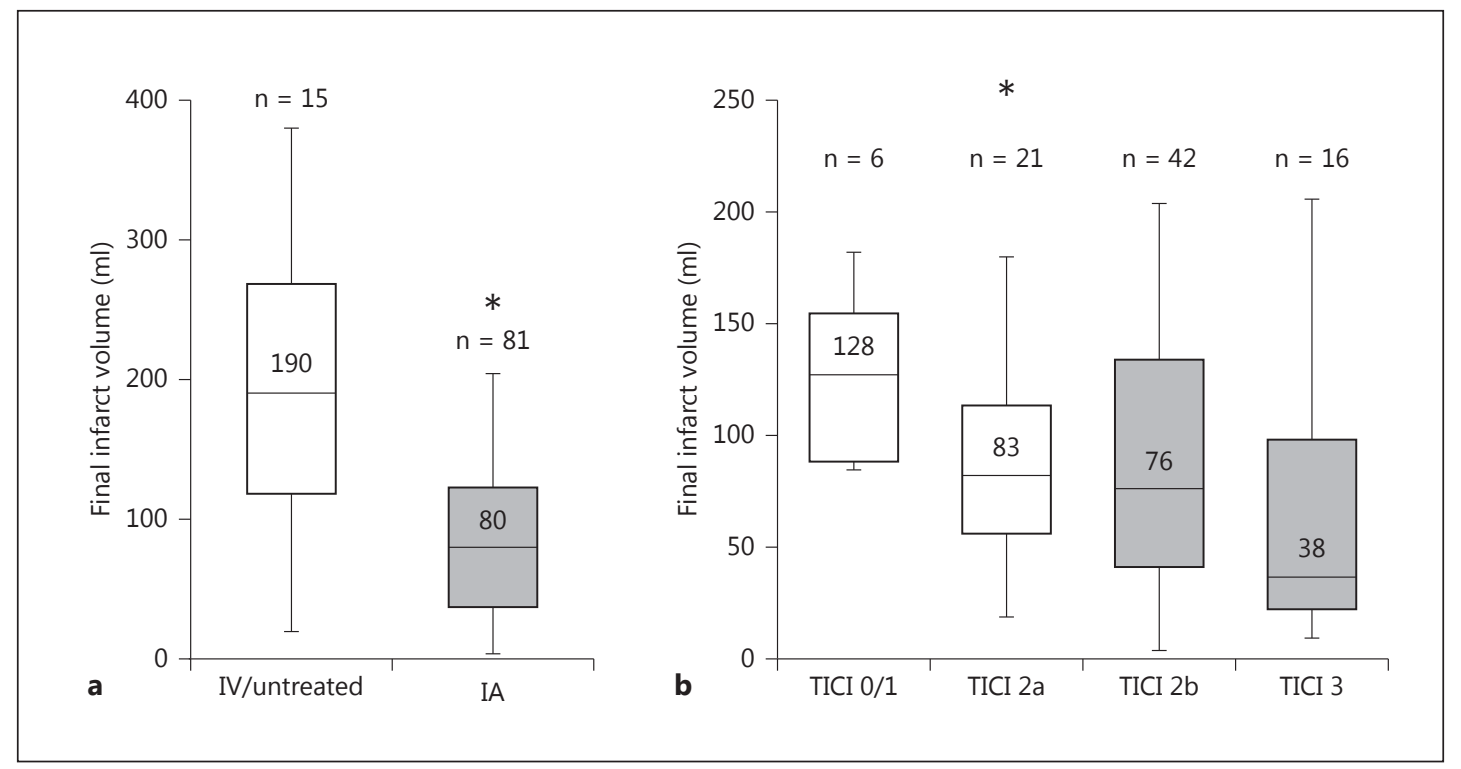

Fig. 2. a Comparison of final infarct volumes in patients treated with IAT versus medical treatment (with or without IV rt-PA) alone $(* \mathrm{p}=0.015)$. b Association between degree of reperfusion (mTICI score) and final infarct volumes $(* \mathrm{p}=0.02)$.

Symptomatic and asymptomatic ICH occurred in $9(10 \%)$ and 31 (36\%) of all IAT patients, respectively. Thirty-seven patients underwent thrombectomy without IV rt-PA. Nine of these patients had acceptable clinical outcome $(24 \%)$ and 8 patients had good clinical outcome $(22 \%)$. The rates of asymptomatic and symptomatic hemorrhage in the IAT patients not treated with IV rt-PA were 32 and 14\%, respectively.

There was no statistically significant difference in mortality in mTICI $0-2$ a versus mTICI $2 b-3$ patients ( 52 vs. $41 \%$; $p=0.5$ ). Rates of good and acceptable clinical outcomes did not differ based on ASPECTS 5, 6 or 7, possibly due to our limited sample size. Time from LKN to intraarterial reperfusion did not correlate with final infarct volume or with 90-day mRS scores.

Impact of Endovascular Therapy versus Medical Treatment Alone in Final Infarct Volumes and Hemicraniectomy Requirements

The median final infarct volume in the IAT group was significantly lower as compared to the medically treated group [fig. 2a; $80 \mathrm{ml}$ (IQR 38-122) vs. $190 \mathrm{ml}(121-267) ; \mathrm{p}=0.015$ ]. Patients with favorable final infarct volume profiles $(<70 \mathrm{ml})$ had a significantly higher probability of achieving good outcome (OR $=0.17,95 \%$ CI $0.05-0.61 ; p=0.004)$. There was a statistically significant correlation between better degrees of reperfusion and lower infarct volumes in the IAT patients (fig. $2 \mathrm{~b} ; \mathrm{p}=0.02$ ). A strong trend towards a higher hemicraniectomy requirement was seen in medically as compared to endovascularly treated patients ( 20 vs. $6 \% ; p=0.06$ ) despite similar in-hospital mortality ( 20 vs. $26.7 \%$; $=0.75$ ).

\section{Discussion}

In addition to using level of occlusion and NIHSS score as selection criteria, a rapid estimation of established infarct using ASPECTS can be reliably used to select patients for endovascular therapy. Using this approach, patients with ASPECTS $>7$ have preferentially received 
endovascular therapy, while data in patients with intermediate ASPECTS 5-7 are more scant. To our knowledge, the current study represents the largest series of patients presenting with LVOS and ASPECTS of 5-7 who have undergone endovascular therapy. In our cohort, we have found that better reperfusion status through endovascular revascularization seems to result in significantly lower final infarct volumes. This may lead to a higher likelihood of better clinical outcomes and may also lessen the need for hemicraniectomy.

An important consideration raised by the current analysis is the need to adjust one's expectations to the individual situation one faces in clinical practice. Traditionally, the initial NIHSS has served as the most important determinant of acute stroke severity. Clinical trial designs are now taking into account risk stratification by using an mRS shift analysis to better adjust outcome targets with initial stroke severity (e.g. NIHSS). From previous work, ASPECTS has been shown to be an independent predictor of outcome. As such, it should be included in risk stratification and adjustment of targets for determining acceptable outcomes. For instance, when treating an LVOS patient with a favorable baseline imaging profile (e.g. ASPECTS >7), it is very reasonable to expect an excellent (90-day mRS 0-1) or good (mRS $0-2$ ) functional outcome. On the other hand, the expectations need to be tailored in the presence of more extensive established ischemic injury on baseline imaging. However, the presence of larger infarct cores on presentation does not necessarily translate into the lack of benefit from reperfusion as such patients may still have a shift towards better functional status. In these cases, achieving an ambulatory status (even if not full independence, mRS $0-3$ ) seems to be a more appropriated outcome measure. Indeed, 90-day mRS 0-3 (and even $0-4)$ has been previously used to establish the benefit of hemicraniectomy in patients presenting with extensive MCA infarcts [17]. To further exemplify, in a patient with ASPECTS 5-7 at risk of subsequent progression to a malignant MCA infarct, it seems counterintuitive that one would reject the opportunity of endovascular reperfusion during an earlier phase of treatment simply because complete independence seems unlikely to be achievable (likely in the setting of significant additional brain tissue loss) to consider a decompressive hemicraniectomy later on in order to achieve a similar or potentially worse functional status as the one that could have been obtained with endovascular reperfusion. In our study, endovascular therapy achieved acceptable outcomes in 33\% of ASPECTS 5-7 patients. Importantly, acceptable outcomes were attained in nearly $45 \%$ of patients who achieved mTICI $2 \mathrm{~b}-3$ reperfusion.

It is important to highlight that the majority of patients in the current study were treated with early-generation devices (e.g. Merci retriever and initial generation Penumbra system) and that both reperfusion and clinical outcomes seem to be better with the use of newer generation devices [18]. It is therefore reasonable to postulate that treatment outcomes in LVOS patients with ASPECTS 5-7 might be better with the use of the newer generation devices now available.

Our results are in agreement with the published outcomes for the ASPECTS 5-7 LVOS in the Interventional Management of Stroke III trial (IMS-III) [19]. In IMS-III, there were a total of 89 patients with ASPECTS of 5-7. Sixty-seven of these received combined IV/IA treatment and the remaining IV rt-PA only. Eighteen of the 89 IMS-III patients (20\%) reached 90 -day mRS of $0-2$, which is again similar to our IAT group. The overall rates of symptomatic and asymptomatic ICH were $9 / 89$ (13\%) and 25/89 (37\%), which are similar to the rates in our endovascular treatment cohort. However, there are two important differences between the IMS-III and the current study cohorts. First, the median time from stroke onset to groin puncture was much longer in our patients ( $202 \mathrm{vs} .300 \mathrm{~min}$ ). Second, only $43 \%$ of our patients received IV tPA in contrast to $100 \%$ of the IMS-III patients. It is therefore remarkable that our patients achieved similar outcomes to IMS-III despite being treated later and having received IV rt-PA in less than half of the IMS-III proportion. We believe this was related to the higher 
Noorian et al.: Endovascular Therapy in Strokes with ASPECTS 5-7 May Result in

rate of mTICI $2 b-3$ reperfusion seen in our study as compared to IMS-III, likely reflecting the higher usage of stent retrievers in our cohort ( 41 vs. $1.5 \%$ ).

Our study has many of the limitations inherent to a retrospective methodology. We also had a relatively small sample size especially for the medically treated group. Moreover, a long-term difference in functional outcomes between IAT and medically treated patients could not be demonstrated since 90-day outcomes were only collected for IAT patients. However, despite our small sample size, we were able to demonstrate that final infarct volumes were significantly smaller in IAT patients and were directly correlated with better degrees of reperfusion. Notably, final infarct volume has been recently established as one of the most powerful surrogates for long-term clinical outcomes [20,21].

In conclusion, despite an overall low probability of achieving functional independence, endovascular therapy in patients with LVOS and ASPECTS 5-7 seems to be safe and may result in smaller infarct sizes, potentially leading to lower degrees of disability and lower rates of requirement for hemicraniectomy. Therefore, endovascular reperfusion in patients with severe stroke symptoms who have midrange ASPECTS on noncontrast CT scan might be a sensible option for patients and families who favor a shift from severe to moderate disability. Due to the inherent limitations of a retrospective cohort study and the lack of a matched control group, prospective studies utilizing a shift in outcome measures are warranted to confirm these results.

\section{Disclosure Statement}

RG Nogueira is Trevo-2 and DAWN Trials Principal Investigator for Stryker Neurovascular, he is also in the SWIFT and SWIFT Prime Trials Steering Committee and the STAR Trial Angiographic Core Lab of Covidien, in the 3D Separator Trial Executive Committee of Penumbra Inc. and is a member of the Stroke Trial DSMB of Rapid Medical. Furthermore, he is Editor-In-Chief of the journal Interventional Neurology. There is no competing interest for the rest of the authors

\section{References}

1 Katzan IL, Hammer MD, Hixon ED, Furlan AJ, Abou-Chebl A, Nadzam DM, et al: Utilization of intravenous tissue plasminogen activator for acute ischemic stroke. Arch Neurol 2004;61:346-350.

2 Minnerup J, Wersching H, Ringelstein EB, Schilling M, Schabitz WR, Wellmann J, et al: Impact of the extended thrombolysis time window on the proportion of recombinant tissue-type plasminogen activator-treated stroke patients and on door-to-needle time. Stroke 2011;42:2838-2843.

$>3$ Lindsberg PJ, Mattle HP: Therapy of basilar artery occlusion: a systematic analysis comparing intra-arterial and intravenous thrombolysis. Stroke 2006;37:922-928.

4 Saqqur M, Uchino K, Demchuk AM, Molina CA, Garami Z, Calleja S, et al: Site of arterial occlusion identified by transcranial Doppler predicts the response to intravenous thrombolysis for stroke. Stroke 2007;38:948-954.

5 Del Zoppo GJ, Poeck K, Pessin MS, Wolpert SM, Furlan AJ, Ferbert A, et al: Recombinant tissue plasminogen activator in acute thrombotic and embolic stroke. Ann Neurol 1992;32:78-86.

6 Bhatia R, Hill MD, Shobha N, Menon B, Bal S, Kochar P, et al: Low rates of acute recanalization with intravenous recombinant tissue plasminogen activator in ischemic stroke: real-world experience and a call for action. Stroke 2010;41:2254-2258.

7 Broderick JP, Palesch YY, Demchuk AM, Yeatts SD, Khatri P, Hill MD, et al: Endovascular therapy after intravenous t-PA versus t-PA alone for stroke. N Engl J Med 2013;368:893-903.

8 Rha JH, Saver JL: The impact of recanalization on ischemic stroke outcome: a meta-analysis. Stroke 2007;38: 967-973.

-9 Lima FO, Furie KL, Silva GS, Lev MH, Camargo EC, Singhal AB, et al: Prognosis of untreated strokes due to anterior circulation proximal intracranial arterial occlusions detected by use of computed tomography angiography. JAMA Neurol 2014;71:151-157.

10 Khalessi AA, Natarajan SK, Orion D, Binning MJ, Siddiqui A, Levy EI, et al: Acute stroke intervention. J Am Col Cardiol 2011;4:261-269.

11 Lansberg MG, Thijs VN, Bammer R, Kemp S, Wijman CA, Marks MP, et al: Risk factors of symptomatic intracerebral hemorrhage after tPA therapy for acute stroke. Stroke 2007;38:2275-2278. 
Noorian et al.: Endovascular Therapy in Strokes with ASPECTS 5-7 May Result in

12 Goyal M, Menon BK, Coutts SB, Hill MD, Demchuk AM, et al: Effect of baseline CT scan appearance and time to recanalization on clinical outcomes in endovascular thrombectomy of acute ischemic strokes. Stroke 2011;42: 93-97.

13 Weir N, Pexman JH, Hill MD, Buchan AM; CASES investigators: How well does ASPECTS predict the outcome of acute stroke treated with IV tPA? Neurology 2006;67:516-518.

14 Understanding Alberta Stroke Program Early CT Score (ASPECTS). http://www.aspectsinstroke.com/ (accessed December 14, 2014).

15 Higashida R, Furlan A, Roberts H, Tomsick T, Connors B, Barr J, et al: Trial design and reporting standards for intra-arterial cerebral thrombolysis for acute ischemic stroke. Stroke 2003;34:e109-e137.

-16 Yoo AJ, Verduzco LA, Schaefer PW, Hirsch JA, Rabinov JD, Gonzalez RG: MRI-based selection for intra-arterial stroke therapy: value of pre-treatment diffusion-weighted imaging lesion volume in selecting patients with acute stroke who will benefit from early recanalization. Stroke 2009;40:2046-2054.

17 Vahedi K, Hofmeijer J, Juettler E, Vicaut E, George B, Algra A, et al: Early decompressive surgery in malignant infarction of the middle cerebral artery: a pooled analysis of three randomised controlled trials. Lancet Neurol 2007;6:215-222.

18 Davalos A, Pereira VM, Chapot R, Bonafe A, Andersson T, Gralla J, et al: Retrospective multicenter study of the Solitaire FR for revascularization for acute ischemic stroke. Stroke 2012;43:2699-2705.

19 Hill MD, Demchuk AM, Goyal M, Jovin TG, Foster LD, Tomsick TA, et al: Alberta Stroke Program early computed tomography score to select patients for endovascular treatment: Interventional Management of Stroke (IMS)-III trial. Stroke 2014;45:444-449.

20 Yoo AJ, Chaudhry ZA, Nogueira RG, Lev MH, Schaefer PW, Schwamm LH, et al: Infarct volume is a pivotal biomarker after intra-arterial stroke therapy. Stroke 2012;43:1323-1330.

-21 Zaidi SF, Aghaebrahim A, Urra X, Jumaa MA, Jankowitz B, Hammer M, et al: Final infarct volume is a stronger predictor of outcome than recanalization in patients with proximal middle cerebral artery occlusion treated with endovascular therapy. Stroke 2012;43:3238-3244. 